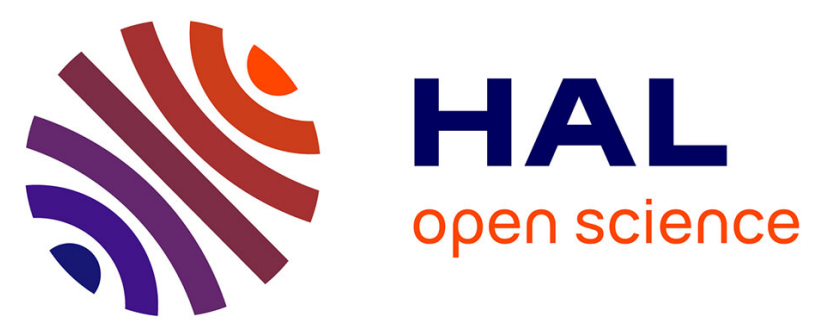

\title{
Modeling Quasi-Periodic Signals by a Non-Parametric Model: Application on Fetal ECG Extraction
}

Saman Noorzadeh, Mohammad Niknazar, Bertrand Rivet, Julie Fontecave-Jallon, Pierre-Yves Guméry, Christian Jutten

\section{- To cite this version:}

Saman Noorzadeh, Mohammad Niknazar, Bertrand Rivet, Julie Fontecave-Jallon, Pierre-Yves Guméry, et al.. Modeling Quasi-Periodic Signals by a Non-Parametric Model: Application on Fetal ECG Extraction. EMBC 2014 - 36th Annual International Conference of the IEEE Engineering in Medicine and Biology Society, Aug 2014, Chicago, United States. hal-01080120

\section{HAL Id: hal-01080120 \\ https://hal.science/hal-01080120}

Submitted on 4 Nov 2014

HAL is a multi-disciplinary open access archive for the deposit and dissemination of scientific research documents, whether they are published or not. The documents may come from teaching and research institutions in France or abroad, or from public or private research centers.
L'archive ouverte pluridisciplinaire $\mathbf{H A L}$, est destinée au dépôt et à la diffusion de documents scientifiques de niveau recherche, publiés ou non, émanant des établissements d'enseignement et de recherche français ou étrangers, des laboratoires publics ou privés. 


\title{
Modeling Quasi-Periodic Signals by a Non-Parametric Model: Application on Fetal ECG Extraction
}

\author{
Saman Noorzadeh ${ }^{1,2}$, Mohammad Niknazar ${ }^{1,2}$, Bertrand Rivet ${ }^{1}$, \\ Julie Fontecave-Jallon ${ }^{2}$, Pierre-Yves Guméry ${ }^{2}$, and Christian Jutten ${ }^{1}$
}

\begin{abstract}
Quasi-periodic signals can be modeled by their second order statistics as Gaussian process. This work presents a non-parametric method to model such signals. ECG, as a quasi-periodic signal, can also be modeled by such method which can help to extract the fetal ECG from the maternal ECG signal, using a single source abdominal channel. The prior information on the signal shape, and on the maternal and fetal RR interval, helps to better estimate the parameters while applying the Bayesian principles. The values of the parameters of the method, among which the R-peak instants, are accurately estimated using the Metropolis-Hastings algorithm. This estimation provides very precise values for the $R$-peaks, so that they can be located even between the existing time samples.
\end{abstract}

\section{INTRODUCTION}

Although fetal electrocardiogram (fECG) may bring useful information about the health of fetus in fetal cardiac monitoring, there is not yet a definite solution to obtain it in a noninvasive manner. This limitation is mainly due to the low signal to noise ratio (SNR) of the fetal ECG recorded from abdominal sensors, which is highly contaminated by the powerful maternal ECG (mECG). The fetal ECG signal recorded from the maternal abdomen is also contaminated by other kinds of sources such as electromyogram (EMG); yet, the most important interference is the maternal ECG.

There exist different approaches for solving the mentioned problem, among which one can name blind source separation methods [1], or adaptive filters [2]. However, these methods require more than one sensor for the data source, while we use one single sensor, since in fetal heart monitoring it would be more convenient and has lower cost. For this purpose, there are a number of methods which have already been examined, such as nonlinear decomposition [3], or shrinkage wavelet denoising [4]. Nevertheless, the most efficient method can be considered as Kalman filtering [5]. However, because of the strong assumption of Kalman filter to model the unobserved state, the authors have already proposed a non-parametric method in order to model the second order statistics of the data using Gaussian process [6], [7]. In this paper, we mainly use the same method, but we will consider other problems in this regard.

This work will investigate the modeling of a quasi-periodic signal like ECG, using a non-parametric model. Here, the problem of estimation of the model parameters is tackled, and using the physiological and mathematical characteristics of the ECG signal [8], [9], a prior information is defined

\footnotetext{
${ }^{1}$ GIPSA-lab, CNRS UMR-5216, Grenoble University, Grenoble, France

${ }^{2}$ TIMC-lab, UMR-CNRS 5525, Joseph Fourier University, Grenoble
}

for the parameters. The problem of fetal R-peak detection is also targeted. Although the detection of maternal R-peak instants is less difficult, the fetal R-peaks could disappear in the presence of EMG, maternal ECG and other powerful interferences and noises. Most of the methods that try to detect fetal R-peaks have been unable to detect these peaks in noisy backgrounds [10], [11]. However, modeling the second order statistics of the data would help to improve the robustness to the additive noise in the non-parametric modeling. Not only the R-peak instants can be detected using this model, but also the method can increase the precision of the detected peaks to be estimated even between the data samples. Therefore, it could let us compensate the missing information if the data does not have enough samples, or if there exists a data loss in the source; for example, the peaks of QRS waveforms could be cut off due to the saturation in amplifier that can be caused by high electrode offset voltage, or improperly calibrated amplifiers. However, the missing samples can be reconstructed according to the nonparametric method.

In section II the non-parametric modeling method is briefly presented to model a quasi-periodic signal. Afterwards the fetal ECG extraction is described in section III as an application of the non-parametric modeling. The proposed method is tested on real data, which are presented in section IV. Section V will sum up the proposed methods and analyses.

\section{NON-PARAMETRIC MODEL FOR A QUASI-PERIODIC SIGNAL}

In this section, the non-parametric modeling of a quasiperiodic signal is presented.

Let $s(t)$ denote the quasi-periodic signal. By considering this signal as a statistical process, it can be described by its second order statistics: i.e. its mean function $m(t) \triangleq$ $\mathbb{E}[s(t)]$ and its covariance function $k\left(t_{1}, t_{2}\right) \triangleq \mathbb{E}\left[\left(s\left(t_{1}\right)-\right.\right.$ $\left.\left.m\left(t_{1}\right)\right)\left(s\left(t_{2}\right)-m\left(t_{2}\right)\right)\right]$ [12]. As a consequence, considering only its second order statistics, it relies among the Gaussian process (GP) framework which is widely used in machine learning e.g., [13], [14] and is denoted by $s(t) \sim$ $\mathcal{G P}\left(m(t), k\left(t_{1}, t_{2}\right)\right)$. Depending on the semidefinite positive function chosen as the covariance one $k(\cdot, \cdot)$ of the GP, it is possible to describe the expected properties of the modeled signal $s(t)$. For instance, the classical square exponential function $k\left(t_{1}, t_{2}\right)=\exp \left(-\left(t_{1}-t_{2}\right)^{2} /\left(2 l^{2}\right)\right)$ allows to model a stationary process whose smoothness is adjusted by the length scale $l$ : the larger the value of $l$ is, the smoother the process is. 


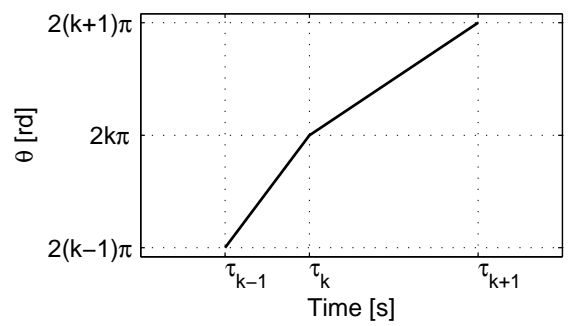

Fig. 1. Illustration of the time warping: each heart beat is linearly wrapped into a $2 \pi$ length interval.

To model an ECG signal as a GP, we propose the following covariance function:

$$
\begin{aligned}
& k\left(t_{1}, t_{2} ; \boldsymbol{\theta}\right)=\sigma^{2} \exp \left(-\frac{\left(t_{1}-t_{2}\right)^{2}}{2 l^{2}}\right) \times \\
& \exp \left(-\frac{\sin \left(\phi\left(t_{1} ;\left\{\tau_{k}\right\}_{k}\right)-\phi\left(t_{2} ;\left\{\tau_{k}\right\}_{k}\right)\right)^{2}}{2 \lambda^{2}}\right),
\end{aligned}
$$

where $\boldsymbol{\theta}=\left\{\sigma^{2}, l^{2}, \lambda^{2},\left\{\tau_{k}\right\}_{k}\right\}$ is the set of hyperparameters, $\left\{\tau_{k}\right\}_{k}$ is the set of R-peak instants and $\phi\left(\cdot ;\left\{\tau_{k}\right\}_{k}\right)$ is a time warping function which models the quasi-periodicity of the ECG as detailed below. This covariance function allows to well fit the properties of an ECG. Indeed, the second term of (1), i.e. $\exp \left(-\sin \left(\phi_{1}-\phi_{2}\right)^{2} /\left(2 \lambda^{2}\right)\right)$ which defines a strictly $\pi$-periodic process, is completed by the time warping function $\phi\left(\cdot ;\left\{\tau_{k}\right\}_{k}\right)$ which wraps each heart beat into an interval of length $2 \pi: \phi\left(t ;\left\{\tau_{k}\right\}_{k}\right)$ is defined such that each interval $\left[\tau_{k-1}, \tau_{k}\right)$ is linearly mapped into the interval $[2(k-1) \pi, 2 k \pi)$ (Fig. 1). An ECG beat mapped to the phase domain is shown in Fig. 2. In other words, the second term in (1) allows to adjust the duration of each beat to model the variability of the RR intervals. Finally, the first term in (1), i.e. $\exp \left(-\left(t_{1}-t_{2}\right)^{2} /\left(2 l^{2}\right)\right)$, models the shape variability of each heart beat by adjusting the correlation between consecutive beats with $l^{2}$. Figure 3 shows two functions drawn at random from a zero-mean GP prior with covariance function (1), $\sigma^{2}=1, l=10$ and $\lambda=0.4$. For these two examples, the two sets of $\tau_{k}$ are different.

Obviously, to correctly model a real ECG, the hyperparameters $\boldsymbol{\theta}$ must be adjusted according to the recorded data. One way is to maximize the log-likelihood:

$$
\hat{\boldsymbol{\theta}}=\arg \max _{\boldsymbol{\theta}} \log p(s(t) \mid \boldsymbol{\theta}),
$$

where $p(s(t) \mid \boldsymbol{\theta})$ is the density probability function (pdf) of $s(t)$ given the parameters $\boldsymbol{\theta}$. If one wants to embed

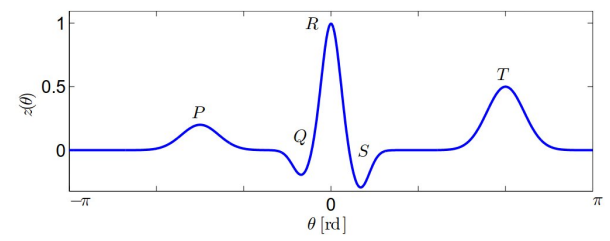

Fig. 2. An ECG beat in phase domain

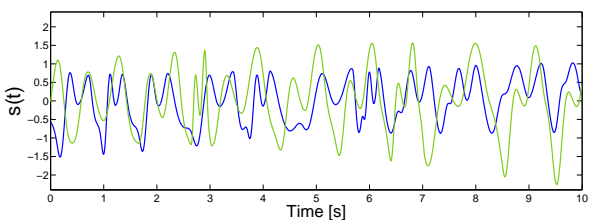

(a) Two randomly drawn priors

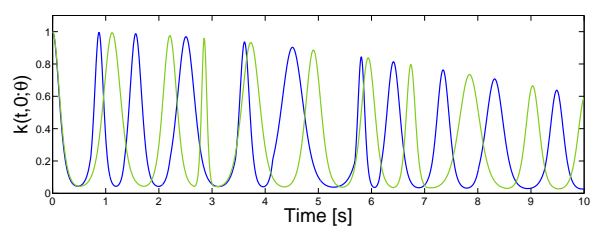

(b) Corresponding covariance functions $k(t, 0 ; \boldsymbol{\theta})$

Fig. 3. Illustration of the flexibility of a GP modeling: two functions randomly drawn from $\mathcal{G P}\left(0, k\left(t_{1}, t_{2}\right)\right)$ with the same covariance function (1) but two different sets of $\tau_{k}$.

some prior knowledge on the signal shape, it could be more advantageous to use a Bayesian scheme by estimating the hyper-parameters $\boldsymbol{\theta}$ as the maximum of the posterior pdf:

$$
\hat{\boldsymbol{\theta}}=\arg \max _{\boldsymbol{\theta}} \log p(\boldsymbol{\theta} \mid s(t)),
$$

where $p(\boldsymbol{\theta} \mid s(t)) \propto p(s(t) \mid \boldsymbol{\theta}) p(\boldsymbol{\theta})$, with $p(\boldsymbol{\theta})$ the prior distribution of the hyper-parameters. In this study, the prior is chosen as a separable term: $p(\boldsymbol{\theta})=p\left(\sigma^{2}\right) p\left(l^{2}\right) p\left(\lambda^{2}\right) p\left(\left\{\tau_{k}\right\}_{k}\right)$, where $p\left(\sigma^{2}\right), p\left(l^{2}\right)$ and $p\left(\lambda^{2}\right)$ are of Gamma pdfs $(\Gamma(\alpha ., \beta$.), with $\left.\cdot \in\left\{\sigma^{2}, l^{2}, \lambda^{2}\right\}\right)$ and $p\left(\left\{\tau_{k}\right\}_{k}\right)$ is derived from the distribution of the RR intervals. By assuming that all the RR intervals are independent and identically distributed with a gaussian pdf of mean $\mu_{R}$ and variance $\sigma_{R}^{2}, p\left(\left\{\tau_{k}\right\}_{k}\right)$ is a multivariate gaussian distribution with mean vector $\mu$, whose $k$-th element is $\tau_{0}+k \mu_{R}$ and covariance matrix $\Sigma$, whose $(i, j)$-th entry is $\Sigma_{i, j}=\min (i, j) \sigma_{R}^{2}$.

\section{APPLICATION TO FETAL ECG EXTRACTION}

In this section, the proposed non-parametric model of ECG signal (Section II) is applied to extract fetal ECG from a single sensor placed on the pregnant woman's abdomen. In this case, one can model the recorded signal $x(t)$ as the summation of the fetal ECG $s_{f}(t)$, the maternal ECG $s_{m}(t)$ and an additive noise $n(t)$ :

$$
x(t)=s_{m}(t)+s_{f}(t)+n(t) .
$$

Both ECG signals are modeled as GP:

$$
\begin{aligned}
s_{m}(t) & \sim \mathcal{G P}\left(0, k_{m}\left(t_{1}, t_{2} ; \boldsymbol{\theta}_{m}\right)\right), \\
s_{f}(t) & \sim \mathcal{G P}\left(0, k_{f}\left(t_{1}, t_{2} ; \boldsymbol{\theta}_{f}\right)\right),
\end{aligned}
$$

with the covariance matrices defined by (1). Moreover, the noise $n(t)$ which is composed of the baseline variations and the remaining noise is also modeled as a zero-mean GP whose covariance function $k_{n}\left(t_{1}, t_{2} ; \boldsymbol{\theta}_{n}\right)$ is defined by

$$
k_{n}\left(t_{1}, t_{2} ; \boldsymbol{\theta}_{n}\right)=\sigma_{b l}^{2} \exp \left(-\frac{\left(t_{1}-t_{2}\right)^{2}}{2 l_{b l}^{2}}\right)+\sigma_{n}^{2} \delta\left(t_{1}-t_{2}\right) \text {, }
$$


where $\boldsymbol{\theta}_{n}=\left\{\sigma_{b l}^{2}, l_{b l}^{2}, \sigma_{n}^{2}\right\}$ and $\delta(\cdot)$ is the delta-Dirac function. The first term corresponds to the baseline (i.e. a smooth signal) and the second one defines a white Gaussian noise. Hence, the posterior estimation of signals $s_{f}(t)$ and $s_{m}(t)$ at time $t_{*}$ are given by

$$
\begin{aligned}
\hat{s}_{m}\left(t_{*}\right) & =\mathbf{k}_{m}\left(t_{*}\right)^{T} K^{-1} \mathbf{x}, \\
\hat{s}_{f}\left(t_{*}\right) & =\mathbf{k}_{f}\left(t_{*}\right)^{T} K^{-1} \mathbf{x},
\end{aligned}
$$

where $\mathbf{x}=\left[x\left(t_{0}\right), \cdots, x\left(t_{N-1}\right)\right]^{T}$ is the vector of the $N$ observed data at time $t_{i}, K$ is the covariance matrix of the observed data whose $(i, j)$-th entry is

$$
K_{i, j}=k_{m}\left(t_{i}, t_{j} ; \hat{\boldsymbol{\theta}}_{m}\right)+k_{f}\left(t_{i}, t_{j} ; \hat{\boldsymbol{\theta}}_{f}\right)+k_{n}\left(t_{i}, t_{j} ; \hat{\boldsymbol{\theta}}_{n}\right),
$$

and $\mathbf{k}_{m}\left(t_{*}\right)=\left[k_{m}\left(t_{*}, t_{0} ; \hat{\boldsymbol{\theta}}_{m}\right), \cdots, k_{m}\left(t_{*}, t_{N-1} ; \hat{\boldsymbol{\theta}}_{m}\right)\right]^{T}$ and $\mathbf{k}_{f}\left(t_{*}\right)=\left[k_{f}\left(t_{*}, t_{0} ; \hat{\boldsymbol{\theta}}_{f}\right), \cdots, k_{f}\left(t_{*}, t_{N-1} ; \hat{\boldsymbol{\theta}}_{f}\right)\right]^{T}$ are the vectors of the covariances evaluated at all pairs of observed $t_{i}$ and estimated times $t_{*}$. The optimal values of the parameters $\hat{\boldsymbol{\theta}}_{m}, \hat{\boldsymbol{\theta}}_{f}, \hat{\boldsymbol{\theta}}_{n}$ are given by the maximization of the posterior of these parameters:

$$
\hat{\boldsymbol{\theta}}=\arg \max _{\boldsymbol{\theta}} p(\boldsymbol{\theta} \mid \mathbf{x})=\arg \max _{\boldsymbol{\theta}} p(\mathbf{x} \mid \boldsymbol{\theta}) p(\boldsymbol{\theta}),
$$

with $\theta=\left\{\boldsymbol{\theta}_{m}, \boldsymbol{\theta}_{f}, \boldsymbol{\theta}_{n}\right\}$ and $p(\boldsymbol{\theta})=p\left(\boldsymbol{\theta}_{m}\right) p\left(\boldsymbol{\theta}_{f}\right) p\left(\boldsymbol{\theta}_{n}\right)$ because the fetal and maternal signals and the noise are considered to be independent. Since this posterior distribution is not trackable in a direct analytic way, it is proposed to sample it using the Metropolis-Hastings algorithm [15] and then to chose the more accurate values of $\boldsymbol{\theta}$.

Several important remarks should be noticed. First of all, the estimated time $t_{*}$ can be any value: it is not restricted to be one of the observed times $t_{i}, i \in\{0, \cdots, N-1\}$. This means that the recorded signals can be down sampled before the estimation of the hyper-parameters by (8) to reduce the computational time. The hyper-parameters $\alpha_{k}$ and $\beta_{k}$ defining the priors are chosen based on physiological analyses to fit a reasonable set of values. The same choice is made for the hyper-parameters $\left\{\mu_{R}, \sigma_{R}\right\}_{k \in\{f, m\}}$ defining the heart rates of mother and fetus [16], [17]. In case of multiple pregnancy, the proposed methods can still be used by adjusting the model (4) with the number of fetuses. Finally, the optimization of the hyper-parameters (8) provides estimations of the maternal and fetal R-peak times by $\left\{\hat{\tau}_{k}^{(m)}\right\}_{k}$ and $\left\{\hat{\tau}_{k}^{(f)}\right\}_{k}$, respectively. It is worth noting that these estimated R-peak times are not limited to the sampled times but can be more precise.

\section{RESULTS}

The proposed method is applied on two data sets. First, the proposed method is used to extract fetal and maternal ECG from a single sensor. Then, the accuracy of the fetal and maternal R-peak detection is investigated.

\section{A. Fetal and maternal ECG extraction}

Based on physiological considerations, the hyperparameters defining the prior Gamma distributions and the normal distribution of the RR intervals of $\boldsymbol{\theta}_{f}$ and $\boldsymbol{\theta}_{m}$ are summarized in Table I.

\begin{tabular}{|l|c|c|}
\hline & Maternal & Fetal \\
\hline$\sigma^{2}\left[\mathrm{~V}^{2}\right]$ & $\Gamma(5, .2)$ & $\Gamma(5, .001)$ \\
\hline$\lambda^{2}[]$ & $\Gamma(5, .005)$ & $\Gamma(5, .002)$ \\
\hline$l^{2}\left[\mathrm{~s}^{-2}\right]$ & $\Gamma(5,40)$ & $\Gamma(5,10)$ \\
\hline$R R$ interval $[\mathrm{s}]$ & $\mathcal{N}(1, .002)$ & $\mathcal{N}(.5, .004)$ \\
\hline
\end{tabular}

TABLE I

VALUES OF HYPER-PARAMETERS OF PRIOR DISTRIBUTIONS ON $\boldsymbol{\theta}_{m}$ AND $\boldsymbol{\theta}_{f}$.

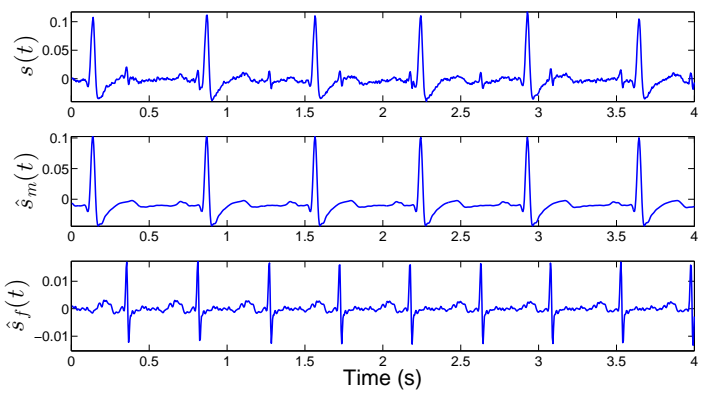

(a) DaISy dataset
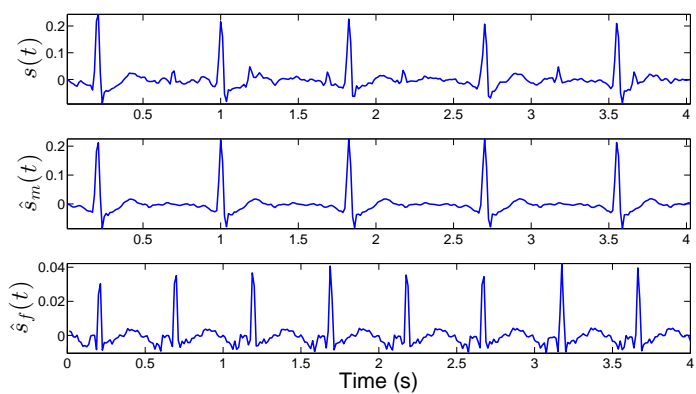

(b) Own dataset

Fig. 4. Illustration of the extraction of fetal ECG. The top plot shows the recorded signal, middle plot shows the extracted maternal ECG and the bottom plot shows the extracted fetal ECG.

The first data is a single-channel abdominal ECG taken from the DaISy dataset [18], with a sampling frequency $f_{s}$ of $250 \mathrm{~Hz}$. The maternal and fetal ECG signals are extracted from this data, and the results are shown in Figure 4(a). The second data is a single-channel abdominal ECG recorded on an 8th month pregnant woman with a sampling frequency of $4 \mathrm{kHz}$ using ADInstruments Bio Amp amplifier, and the Powerlab hardware. As explained in Section III, the data were down sampled to $80 \mathrm{~Hz}$ before applying the proposed method. As one can see, the proposed method is effective to extract fetal ECG even if its amplitude is quite smaller than the maternal ECG. Moreover, the proposed method allows to extract fetal beat even when it arises during the maternal QRS complex (e.g., the first beat on Figure 4(b)).

\section{B. R-peaks detection accuracy}

To show the precision of the method in R-peak detection, our own recorded data is used. The fetal and maternal $\mathrm{R}$ peaks are manually indexed using the original sampling frequency $f_{s}(4 \mathrm{kHz})$ leading to the ground truth of $\mathrm{R}$ peaks times with thus a precision of $250 \mu \mathrm{s}$. The sampling rate 


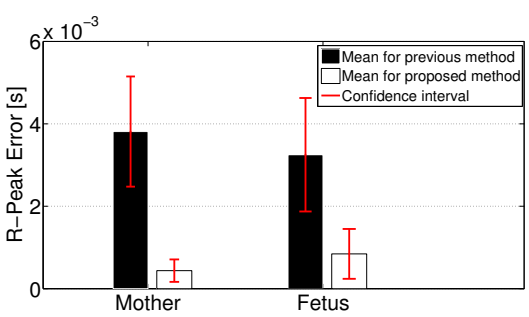

Fig. 5. Accuracy of the R-peaks detection using the proposed method.

of the signal is then reduced by a factor of 50 , and the non-parametric method would extract the fetal and maternal ECG signals from the new signal with the reduced sampling frequency $(80 \mathrm{~Hz})$. The error of R-peak times detected from the extracted ECG signals, comparing the original R-peak times, detected from the original signal, is averaged over all R-peaks and is shown as black bars in Fig. 5 for both maternal and fetal signals. As it was explained before, the R-peak times, as one of the hyper-parameters of the method, can be detected on any time instants ignoring the existing time samples on the signal, so these R-peak time values are estimated using the method from the down-sampled signal, and their mean error comparing the original R-peak times is also depicted in Fig. 5 as white bars. From this figure, which also shows the confidence interval with the confidence level of $99 \%$, it is evident that the estimated samples for R-peaks have a very low error comparing the R-peaks of the downsampled data. In other words, the R-peak times are precisely reconstructed from the signal with a low number of samples.

\section{CONCLUSIONS}

We have studied a non-parametric modeling of quasiperiodic signals, so that they are modeled with Gaussian process (GP) by considering appropriate mean and covariance functions. ECG, as a quasi-periodic signal, is also modeled as GP both for mother and fetus, with mean functions equal to zero and their corresponding covariance functions. The hyper-parameters defining the covariance functions are then estimated accurately by assuming that they follow Gamma prior distributions whose parameters are chosen according to physiological considerations and so that they can describe the shape of the ECG signal. The R-peak instants can also be considered as a set of parameters such that the RR intervals follow a normal distribution. The mean and variance of these normal distributions are also defined to match the statistical behavior of RR intervals. Afterwards, the estimated parameter values, using Metropolis-Hastings algorithm, provide covariance functions that can best describe the maternal and fetal ECG signals. Besides, the R-peaks can be detected at any time instants ignoring the existing time samples of the data. Therefore, even if the signal does not have enough samples, or there is a data loss in the source, the method can reconstruct the missing samples. Keeping in mind that the signals can be extracted at any time (observed time samples or not), this method can be applied on the signal that has been down sampled (i.e., with reduced number of samples), in order to increase the speed of the method.

\section{ACKNOWLEDGMENT}

This work has been supported by the LabEx PERSYVALLab (ANR-11-LABX-0025) and ERC AdG-2012-320864CHESS. The study was also approved by the appropriate local authority (Comit de Protection des Personnes Sud-Est). Data have been acquired with the help of the local facility (PRETA Platform) which belongs to the LSI Carnot Institute Platform Network, and benefits from an official authorization for noninvasive recordings and an open biomedical research protocol both supported by the local university hospital.

\section{REFERENCES}

[1] L De Lathauwer, B De Moor, and Joos Vandewalle, "Fetal electrocardiogram extraction by blind source subspace separation," Biomedical Engineering, IEEE Transactions on, vol. 47, no. 5, pp. 567-572, 2000.

[2] Earl R Ferrara and Bernard Widraw, "Fetal electrocardiogram enhancement by time-sequenced adaptive filtering," Biomedical Engineering, IEEE Transactions on, , no. 6, pp. 458-460, 1982.

[3] M Richter, T Schreiber, D T Kaplan, et al., "Fetal ECG extraction with nonlinear state-space projections," Biomedical Engineering, IEEE Transactions on, vol. 45, no. 1, pp. 133-137, 1998.

[4] F Mochimaru, Y Fujimoto, and Y Ishikawa, "Detecting the fetal electrocardiogram by wavelet theory-based methods," Progress in Biomedical Research, vol. 7, pp. 185-193, 2002.

[5] M. Niknazar, B. Rivet, and C. Jutten, "Fetal ECG extraction by extended state Kalman filtering based on single-channel recordings," Biomedical Engineering, IEEE Transactions on, vol. 60, no. 5, pp. $1345-1352,2013$

[6] B. Rivet, M. Niknazar, and C. Jutten, "Nonparametric modeling of ECG: Applications to denoising and to single sensor fetal ECG extraction," in Proc. of LVA/ICA, 2012, pp. 470-477.

[7] M Niknazar, B Rivet, and C Jutten, "Fetal ECG extraction from a single sensor by a non-parametric modeling," in Signal Processing Conference (EUSIPCO), 2012 Proceedings of the 20th European. IEEE, 2012, pp. 949-953.

[8] Nicholas P Hughes, Lionel Tarassenko, and Stephen J Roberts, "Markov models for automated ECG interval analysis.," in NIPS, 2003.

[9] $\mathrm{H}$ Gholam-Hosseini and $\mathrm{H}$ Nazeran, "Detection and extraction of the ECG signal parameters," in Engineering in Medicine and Biology Society, 1998. Proceedings of the 20th Annual International Conference of the IEEE. IEEE, 1998, pp. 127-130.

[10] C HL Peters, E DM ten Broeke, P Andriessen, B Vermeulen, R CM Berendsen, P FF Wijn, and S Guid Oei, "Beat-to-beat detection of fetal heart rate: Doppler ultrasound cardiotocography compared to direct ECG cardiotocography in time and frequency domain," Physiological Measurement, vol. 25, no. 2, pp. 585, 2004.

[11] E C Karvounis, M G Tsipouras, D I Fotiadis, and K K Naka, "An automated methodology for fetal heart rate extraction from the abdominal electrocardiogram," Information Technology in Biomedicine, IEEE Transactions on, vol. 11, no. 6, pp. 628-638, 2007

[12] A. Papoulis, Probability, Random Variables, and Stochastic Processes, McGraw-Hill, third edition, 1991.

[13] D. J. C. MacKay, "Introduction to Gaussian processes," Neural Networks and Machine Learning, vol. 168, pp. 1-32, 1998.

[14] C. E. Rasmussen and C. K. I. Williams, Gaussian Processes for Machine Learning, MIT Press, 2006.

[15] Dani Gamerman and Hedibert F. Lopes, Markov Chain Monte Carlo, Chapman \& Hall/CRC, 2006.

[16] U Rajendra Acharya, K Paul Joseph, N Kannathal, Choo Min Lim, and Jasjit S Suri, "Heart rate variability: a review," Medical and Biological Engineering and Computing, vol. 44, no. 12, pp. 1031-1051, 2006.

[17] P Van Leeuwen, S Lange, H Bettermann, D Grönemeyer, and W Hatzmann, "Fetal heart rate variability and complexity in the course of pregnancy," Early Human Development, vol. 54, no. 3, pp. 259-269, 1999.

[18] B De Moor, P De Gersem, B De Schutter, and W Favoreel, "Daisy: A database for identification of systems," JOURNAL A, vol. 38, pp. 4-5, 1997. 